\title{
Laparoscopic percutaneous extraperitoneal closure with peritoneum reinforcement repair for pediatric inguinal hernia: a single-center experience with over 2,000 patients
}

\author{
Shouxing Duan", Peijian Zhang", Xiaobin Lin, Lian Zheng \\ Department of Pediatric Surgery, The First Affiliated Hospital of Shantou University Medical College, Shantou, China \\ Contributions: (I) Conception and design: S Duan, P Zhang, L Zheng; (II) Administrative support: S Duan, L Zheng; (III) Provision of study materials \\ or patients: P Zhang, X Lin; (IV) Collection and assembly of data: P Zhang, X Lin; (V) Data analysis and interpretation: S Duan, P Zhang; (VI) \\ Manuscript writing: All authors; (VII) Final approval of manuscript: All authors. \\ \#These authors contributed equally to this work. \\ Correspondence to: Prof. Lian Zheng. Department of Pediatric Surgery, The First Affiliated Hospital of Shantou University Medical College, Shantou \\ 515041, China. Email: lzheng1585@163.com.
}

\begin{abstract}
Background: Inguinal hernia is one of the common diseases in infants and children that requires operative treatment. Laparoscopic inguinal hernia repair in children has become an alternative to the open procedure. Laparoscopic percutaneous extraperitoneal closure with peritoneum reinforcement (LPECPR) is a safe and effective approach for pediatric inguinal hernia, and has a lower recurrence. This is a retrospective study to present our experience with children who underwent LPECPR.
\end{abstract}

Methods: A total of 2,018 patients with inguinal hernia who underwent LPECPR in our hospital from July, 2011 to December, 2020 were reviewed. The surgical technique is modified on the basis of laparoscopic percutaneous extraperitoneal closure (LPEC) to close extraperitoneally by circuit suturing twice around the internal inguinal ring.

Results: All cases were completed LPECPR without conversion. There were no intraoperative complications. A total of 2,018 patients' laparoscopic procedures were achieved. The mean operative time was 14 and $20 \mathrm{~min}$ for unilateral and bilateral operations, respectively. Follow-up to date is 13.4 months (6-36 months), there were no postoperative complications, such as knot reactions, hydrocele formation, testicular atrophy or pain, except 3 recurrences $(3 / 2,018,0.15 \%)$.

Conclusions: This modified LPECPR technique can acquire lower recurrence rate for repair pediatric inguinal hernia. The midterm safety and efficacy of LPECPR are proven and it can be a routine procedure.

Keywords: Inguinal hernia; laparoscopic; peritoneum reinforcement; pediatric

Submitted Jan 15, 2021. Accepted for publication Mar 18, 2021.

doi: $10.21037 / \mathrm{tp}-21-25$

View this article at: http://dx.doi.org/10.21037/tp-21-25

\section{Introduction}

Inguinal hernia is one of the most common pediatric diseases that requires operative treatment (1-3). Laparoscopic repair for pediatric inguinal hernia has become more and more popular (4-6). Laparoscopic percutaneous extraperitoneal closure (LPEC) of the internal ring is a safe and effective approach to pediatric inguinal hernia, and has been widely used with many advantages, such as repair of contralateral patent processus vaginalis (CPPV), low rate of recurrence, reduction of operative time, and good result of cosmesis $(7,8)$.

In this study, we modified a kind of peritoneum reinforcement technique for the repair of pediatric inguinal hernia, which has a lower recurrence rate than LPEC. LPEC with peritoneum reinforcement (LPECPR) circuited and demixed suturing twice around the internal inguinal 
Table 1 Demographic data of all patients

\begin{tabular}{|c|c|}
\hline Parameter & Data (number) \\
\hline Sex & $\begin{array}{l}\text { Males }(1,603) \\
\text { females }(415)\end{array}$ \\
\hline Median age (range), years & $3.27(0.25-15.00)$ \\
\hline \multicolumn{2}{|l|}{ Presentation, n (\%) } \\
\hline Right sided hernia & $1,230(60.95)$ \\
\hline Left sided hernia & $480(23.79)$ \\
\hline Bilateral hernia & $305(15.11)$ \\
\hline Recurrent hernia & $3(0.15)$ \\
\hline Contralateral patent processus vaginalis & $1,074(53.22)$ \\
\hline \multicolumn{2}{|l|}{ Operative time [range], min } \\
\hline Unilateral hernia (initial or recurrent) & $14[7-18]$ \\
\hline Bilateral hernia & 20 [10-35] \\
\hline Males & 19 [15-35] \\
\hline Females & $13[10-20]$ \\
\hline Postoperative hospital stay [range], hours & $<24$ [13-24] \\
\hline \multicolumn{2}{|l|}{ Complications, n (\%) } \\
\hline Recurrence & $3(0.15)$ \\
\hline Scrotal edema & 0 \\
\hline Suture site abscess & 0 \\
\hline Umbilical hernia & 0 \\
\hline latrogenic cryptorchidism & 0 \\
\hline Testicular atrophy & 0 \\
\hline
\end{tabular}

ring to reinforce peritoneum. In the present study, we reviewed the outcomes of 2,018 patients with symptomatic inguinal hernia undergoing LPECPR repair in our center to assess the technical feasibility and efficiency.

We present the following article in accordance with the STROBE reporting checklist (available at http://dx.doi. org/10.21037/tp-21-25).

\section{Methods}

\section{Clinical data of patients}

We retrospectively reviewed 2,018 patients with a markedly inguinal hernia, who underwent LPECPR repair at the department of pediatric surgery of the First Affiliated Hospital of Shantou University Medical College in China between July 2011 and December 2020. The diagnoses were based on clinical presentation and physical examination, with or without ultrasonography. Inclusion criteria for LPECPR repair were as follows: all patients with inguinal hernia diagnosed by non-emergency and patients with emergency incarcerated hernia who could be manual reposition. Exclusion criteria: (I) hernia repair surgery requiring transfer to open surgery; (II) hernia repair in cryptorchidism surgery; (III) hernia repair in appendicitis surgery; (IV) neonatal hernia repair. The main outcome observations of this study included; age, operative time, hospital stay, presence of CPPV, hernia recurrence, scrotal edema, suture site abscess, umbilical hernia, testicular atrophy and iatrogenic ascent of the testis. The patient characteristics are shown in Table 1. This study was approved by the ethics committee of the First Affiliated Hospital of Shantou University Medicine College (B-2021021). All procedures performed in this study involving human participants were in accordance with the Declaration of Helsinki (as revised in 2013). Individual consent for this retrospective analysis was waived.

\section{Homemade hernia needle with Kirschner wire}

A Kirschner wire (Figure 1A), flatten its tip, then punch an oval hole in the center of the front, and finally polish the front of the needle (including the hole) (Figure 1B,C). The end of the needle can be folded into a triangle/oval or untreated. The method of making homemade hernia needle is simple, economical (even cost-free), and effective. It is very suitable for basic hospitals and units with difficulties in purchasing medical instruments.

\section{Surgical procedure}

After general anesthesia, the patients lie supine in the Trendelenburg position at a $15^{\circ}$ tilt. The television screen was placed in the lower right corner of the patient. The surgeon stood at the patient's left while the assistant held the laparoscope at the right side. A 5-mm curvilinear supraumbilical skin incision was made. A Veress needle was inserted into the abdominal cavity, and pneumoperitoneum was established to a pressure of 8 to $10 \mathrm{mmHg}$. A $5-\mathrm{mm}$ trocar was inserted through this incision, and a $5-\mathrm{mm} 30^{\circ}$ laparoscope was inserted to this trocar. A 3-mm stab incision was made on the left of umbilicus for the inserting of a $3-\mathrm{mm}$ trocar. Through the 3-mm trocar, a 3-mm assisted forceps was introduced into the peritoneal cavity. A $1.0-\mathrm{mm}$ stab 


\section{A}

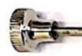

B

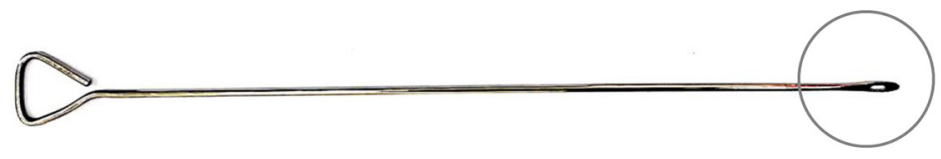

C

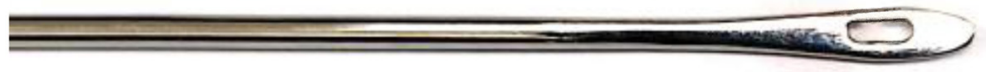

Figure 1 Homemade needle with Kirschner wire for laparoscopic repair inguinal hernias. (A) A Kirschner wire used in orthopedics. (B) Flatten Kirschner wire tip, then punch an oval hole in the center of the front, and finally polish the front of the needle (including the hole). (C) Enlargement of the homemade needle tip (circled) of (B).

incision was made at the surface projection of the internal ring. Under laparoscopic monitoring, a homemade needle with 2-0 polyester nonabsorbable braided suture and 4-0 vicryl suture was inserted into the anterior abdominal wall through the $1.0-\mathrm{mm}$ stab incision (Figure $2 A$ ). The internal half circle was begun extraperitoneally from the internal edge to the external edge around the internal inguinal ring. After the needle advanced on the vas deferens and vessels, or down to the round ligament of uterus, peritoneum was punctured (Figure 2B). The 2-0 suture was released into the abdominal cavity (Figure $2 C$ ). After the needle was withdrawn to the roof of the ring under the peritoneum, it was advanced along the external side of the ring and got into the abdominal cavity through the same puncture hole as the internal half circuit (Figure 2C,D). The 4-0 suture was pulled on one side of the needle to form a loop, and the end of the 2-0 suture was grasped to pass through the loop (Figure 2D). The needle was drawn out of the abdominal cavity. Simultaneously, the 4-0 suture was pulled by the assistant extracorporeally. After this step, the 2-0 suture was placed around the inguinal ring under the peritoneum (Figure $2 E$ ) and both of its ends were passed through the same puncture point and tunnel. After gas in the distal sac was squeezed out, the 2-0 suture was tied tightly extracorporeally, and the internal ring was completely closed. The knot was placed underneath peritoneally.
Through the same $1.0-\mathrm{mm}$ stab incision, a homemade needle with the same sutures was inserted into the anterior abdominal wall about $3-\mathrm{mm}$ far from the initial complete circuit ligation (Figure 3A). Similarly, the 2-0 suture was placed around the inguinal ring under the peritoneum (Figure 3B). Both ends were passed through the same puncture point but via different tunnels. The 2-0 suture was tied tightly extracorporeally, and the knot was placed underneath peritoneally. The second knot was about 3-mm far from the first knot. When asymptomatic CPPV was observed, LPEC repair was performed.

\section{Statistical analysis}

Measurement data and enumeration data were presented as median and $\mathrm{n}(\%)$, respectively. Statistical analyses were done using the SPSS software (version 22.0).

\section{Results}

All patients underwent LPECPR without conversion. They were 1,603 males and 415 females. Their median age was 3.27 years ( 3 months -15 years). The median operation time was $19 \mathrm{~min}(15-35 \mathrm{~min})$ and $13 \mathrm{~min}(10-20 \mathrm{~min})$ for males and females, and $14 \mathrm{~min}(7-18 \mathrm{~min})$ and $20 \mathrm{~min}$ (10-35 $\mathrm{min})$ for unilateral and bilateral inguinal hernias. 

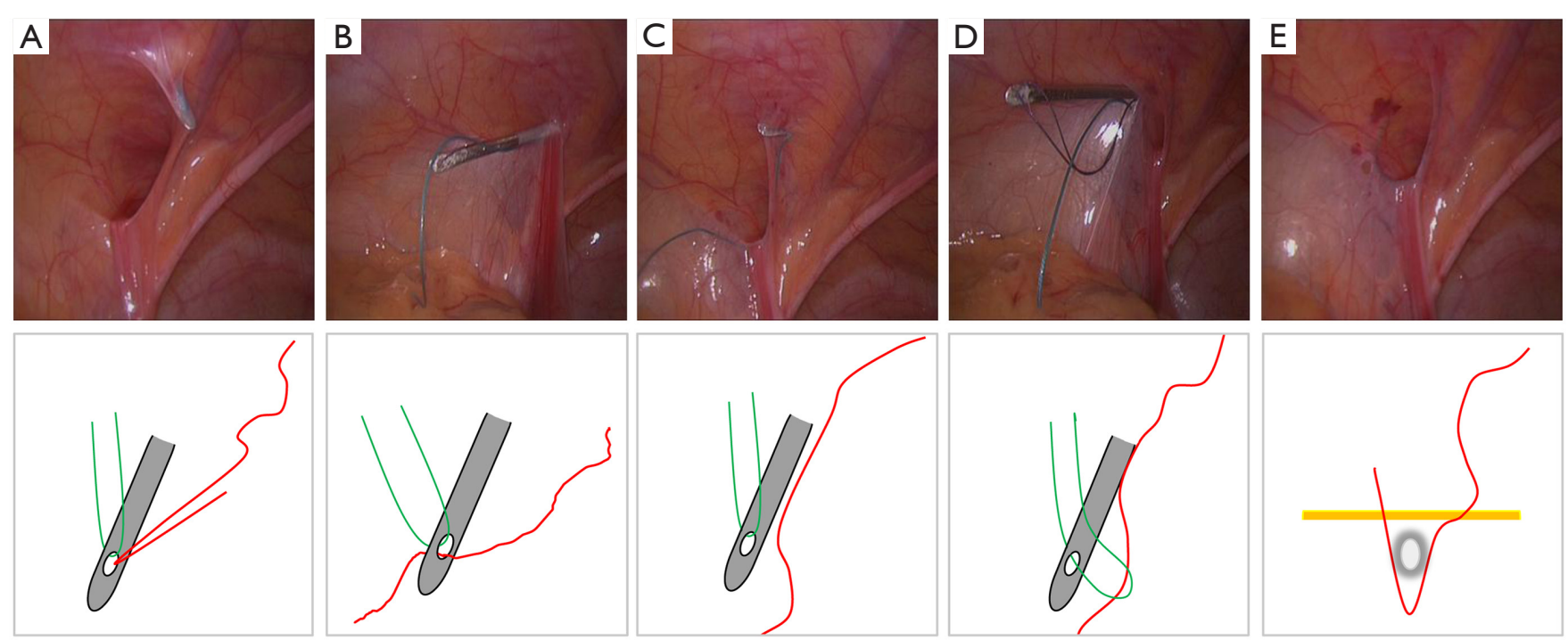

Figure 2 Laparoscopic percutaneous extraperitoneal closure procedure. (A) A homemade needle with sutures was inserted into the anterior abdominal wall extraperitoneal. (B) The internal half circle was begun extraperitoneally from the internal edge to the external edge around the internal inguinal ring, peritoneum was punctured and the suture was released into the abdominal cavity. (C) The needle was withdrawn to the roof of the ring under the peritoneum but the suture was remained. (D) The needle was advanced along the external side of the ring and got into the abdominal cavity through the same puncture hole, the auxiliary suture (green) was pulled on one side of the needle to form a loop, and the end of the closure suture (red) was grasped to pass through the loop. (E) The closure suture was placed around the inguinal ring under the peritoneum. The lower panel was the schematic drawing of the upper panel. The red line was the closure suture and the green line was the auxiliary suture.

There were no intraoperative complications. All patients were discharged within 24 hours after the operation. All patients received regular follow-up examinations, which included physical examination or ultrasonography. The median follow-up period was 13.4 months (6-36 months). There were no postoperative complications, such as knot reactions, hydrocele formation, testicular atrophy and pain, except 3 recurrences $(3 / 2,018,0.15 \%)$. In the three recurrent patients, two school-age boys participated in strenuous exercise after surgery, including one 7-yearold boy, who began to practice taekwondo 3 months after surgery (at least once a week), and hernia recurred 1 month later. Another 9-year-old boy, who began to participate in table tennis practice (twice a week) 2 months after surgery, developed a hernia recurrence 3 months later. The last case, a 2-year-old boy, had a respiratory infection 3 months after surgery and persistent cough for 1 month, and a hernia recurrence. All 3 recurrent patients with recurrence underwent the reoperation with the same approach (LPECPR). All of them were followed up for 3 years and found no recurrence.

\section{Discussion}

Pediatric inguinal hernia repair is a common surgical procedure. Laparoscopic pediatric inguinal hernia repair has become an alternative procedure to open herniotomy (1). Laparoscopic pediatric inguinal hernia repair has been performed with several methods and techniques, including intracorporeal suturing and extracorporeal closure of the internal ring (4). Among all the methods and techniques, LPEC repair is one of mature technology of them $(7,8)$. Compared with conventional open herniotomy, LPEC repair has many advantages such as repair of $\mathrm{CPPV}$, low rate of recurrence, reduction of operative time and good result of cosmesis (7-9). LPEC has rare complications, but recurrence still existed.

Recurrence is the most important problem in patients because they should be required reoperation. The incidence of recurrence has been reported differ. Various risk factors of recurrence have been reported: patient's sex and age, surgeon's experience, use of absorbable sutures, loosening of the knot and leaving a peritoneal gap in the encircling 

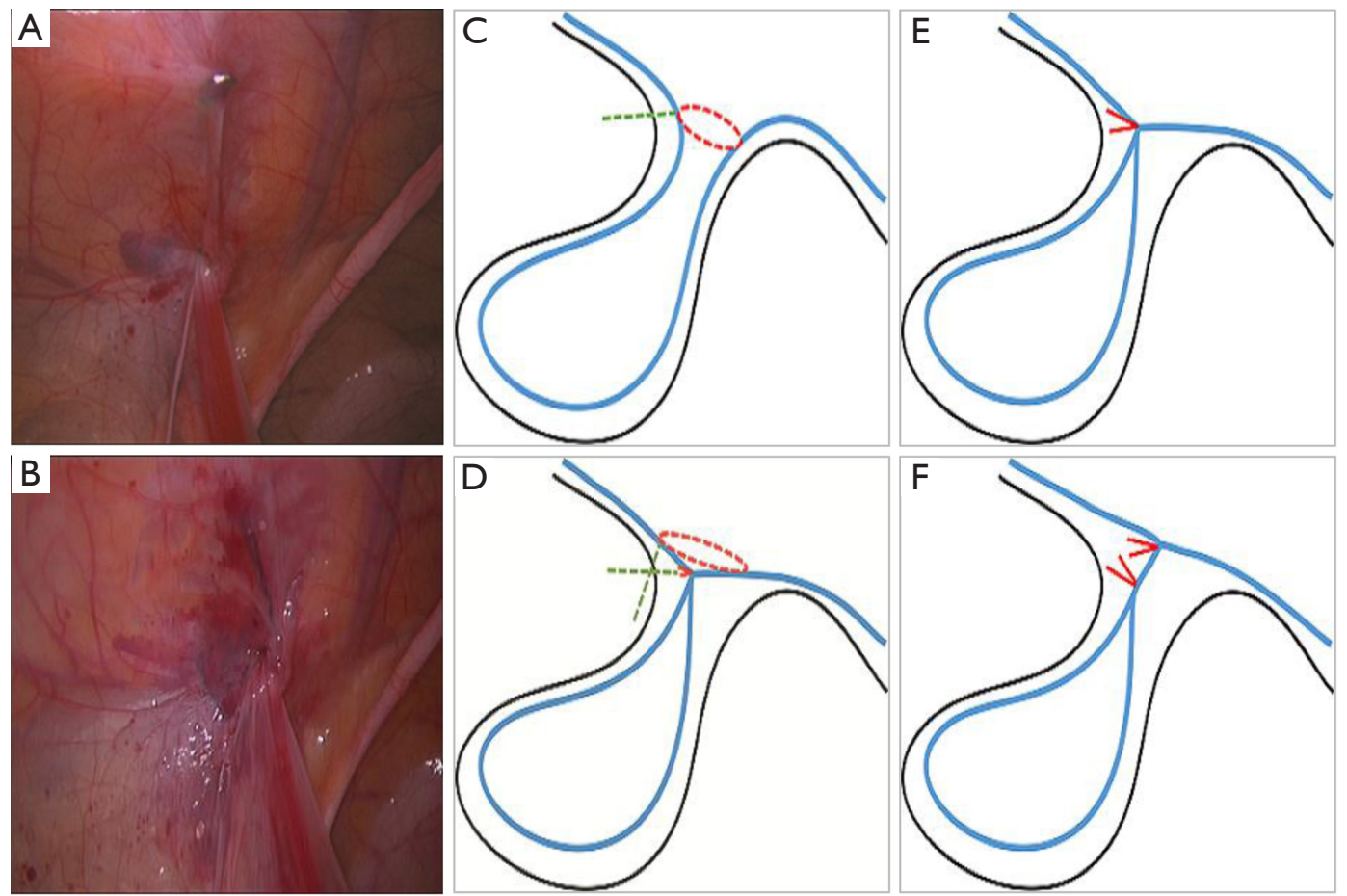

Figure 3 Peritoneum reinforcement of the Laparoscopic percutaneous extraperitoneal closure (LPEC) repair. (A) A homemade needle with the same sutures was inserted into the anterior abdominal wall extraperitoneal about $3 \mathrm{~mm}$ far from the initial complete circuit ligation. (B) The second suture was placed around the inguinal ring under the peritoneum, circuited and demixed suturing twice around the internal inguinal ring to reinforce peritoneum. (C,E) The schematic drawing of (A): (C) the green dotted line: a homemade needle with sutures was inserted into the anterior abdominal wall. The red dotted line: closure of the internal ring; (E) the red line: the knot was placed underneath peritoneally. (D,F) The schematic drawing of (B): (D) the two green dotted lines: a homemade needle with sutures was inserted again into the anterior abdominal wall through the same puncture point but via different tunnels. The red dotted line: closure of the internal ring for peritoneum reinforcement; (F) the red lines: the second knot was placed underneath peritoneally about 3 mm far from the first knot.

suture (10-13). Lee et al. hypothesized that high stretchiness around the internal ring and increased tension on the suture were risk factors of recurrence (14). Hayashi et al. suggested that torn peritoneum and use of single ligation were two preventable factors for recurrence (15). Complete circuit ligation of the hernia sac is important to prevent recurrence.

Experts are looking for a better method to reduce recurrence (16-18). Therefore, we modified the LPEC technique by reinforce peritoneum with extraperitoneal double complete circuit ligation. LPECPR is circuited and demixed suturing twice around the internal inguinal ring to reinforce peritoneum. In this technique, peritoneal reinforcement is performed by stagger closing the internal inguinal ring twice in different planes (Figure $3 C, D$ ). Because the planes are not at the same level, the forces on the two closed planes are not in the same direction (Figure $3 E, F)$. This procedure provides two lines of defense for the closure of the peritoneum, it can reduce or decompose the tension around the internal inguinal ring caused by abdominal pressure. In addition, peritoneum reinforcement can reduce the tension of the first suture and prevent avulsion of the peritoneum, especially in patients with brittleness or edema peritoneum. In the present study, 2,018 patients underwent LPECPR technology at a recurrence rate of $0.15 \%(3 / 2,018)$, lower than the recurrence rate of Miyake et al. $(0.27 \%$, 3/1,109), who also had over 1,000 cases of pediatric hernia LPEC repair surgery experience (7). The low recurrence rate may be related to the peritoneum reinforcement of the LPECPR.

In the present series, we did not have postoperative complications such as scrotal edema, suture site abscess, umbilical hernia, iatrogenic cryptorchidism, or testicular atrophy, except for a very small recurrence. Interestingly, recurrence was seen only in males and within half a year 
after surgery in the three recurrent patients, and they had different risk factors respectively. Two school-age boys participated in strenuous exercise after surgery, with violent pulling and increased abdominal pressure. The last case, another infant had increased abdominal pressure for a long time after surgery. We found that one of the common features of these patients with recurrence was that they all had increased abdominal pressure and/or violent pull as risk factors shortly after surgery $(13,19)$. These recurrent patients were followed up for another 3 years after reoperation. After 1 year of reoperation, the children continued their original training (table tennis practice and taekwondo practice) with no recurrence. Therefore, early surgery is recommended for symptomatic patient with inguinal hernia and risk factors such as increased abdominal pressure should be avoided after surgery.

The homemade hernia needle method we describe is simple, economical, and even free. The unit with laparoscopic equipment can perform such operation with the homemade hernia needle. For pediatric surgeons who are not experienced in this technique, we recommend as follows: a two-port technique was initially used, with one port for laparoscopy and the other for grasping forceps, which is similar to that described previously. Grasping forceps not only makes intraperitoneal handling easier, but also helps the needle pass through the preperitoneal space without skipping over any area where sutures are placed so that the inguinal ring is totally encircled. The key and difficulty of this technique is that the hernia needle avoids the vas deferens and the blood vessels and passes through the preperitoneal space to ensure that the vas deferens or spermatic vessels are not ligated.

\section{Conclusions}

In conclusion, the most technical refinements and modifications of this modified technique can achieve a lower recurrence rate. In addition, this operation is simple, less complications, and cost-effective (it can be homemade surgical instrument at no cost). LPECPR is an efficient and safe method for pediatric inguinal hernia.

\section{Acknowledgments}

Funding: This study was supported by grants from the National Natural Science Foundation of China (grant No.
81801432), the Natural Science Foundation of Guangdong Province of China (grant No. 2018A030307045), the Postdoctoral Science Foundation of China (grant No. 2019M652990) and the "Dengfeng Project" for the construction of high-level hospitals in Guangdong Province-the First Affiliated Hospital of Shantou University Medical College Supporting Funding (grant No. [2019] 70).

\section{Footnote}

Reporting Checklist: The authors have completed the STROBE reporting checklist. Available at http://dx.doi. org/10.21037/tp-21-25

Data Sharing Statement: Available at http://dx.doi. org/10.21037/ tp-21-25

Conflicts of Interest: All authors have completed the ICMJE uniform disclosure form (available at http://dx.doi. org/10.21037/tp-21-25). All authors received payments from the National Natural Science Foundation of China, the Natural Science Foundation of Guangdong Province of China, the Postdoctoral Science Foundation of China, and the "Dengfeng Project" for the construction of highlevel hospitals in Guangdong Province-the First Affiliated Hospital of Shantou University Medical College Supporting Funding.

Ethical Statement: The authors are accountable for all aspects of the work in ensuring that questions related to the accuracy or integrity of any part of the work are appropriately investigated and resolved. The study was conducted in accordance with the Declaration of Helsinki (as revised in 2013). The study was approved by the Ethics Committee at the First Affiliated Hospital of Shantou University Medicine College (B-2021-021) and individual consent for this retrospective analysis was waived.

Open Access Statement: This is an Open Access article distributed in accordance with the Creative Commons Attribution-NonCommercial-NoDerivs 4.0 International License (CC BY-NC-ND 4.0), which permits the noncommercial replication and distribution of the article with the strict proviso that no changes or edits are made and the original work is properly cited (including links to both the formal publication through the relevant DOI and the license). 
See: https://creativecommons.org/licenses/by-nc-nd/4.0/.

\section{References}

1. Esposito C, St Peter SD, Escolino M, et al. Laparoscopic versus open inguinal hernia repair in pediatric patients: a systematic review. J Laparoendosc Adv Surg Tech A 2014;24:811-8.

2. Juang D, Fraser JD, Holcomb GW 3rd. The laparoscopic approach for repair of indirect inguinal hernias in infants and children. Transl Pediatr 2016;5:222-6.

3. Kantor N, Travis N, Wayne C, et al. Laparoscopic versus open inguinal hernia repair in children: which is the true gold-standard? A systematic review and meta-analysis. Pediatr Surg Int 2019;35:1013-26.

4. Esposito C, Escolino M, Farina A, et al. Two decades of history of laparoscopic pediatric inguinal hernia repair. J Laparoendosc Adv Surg Tech A 2014;24:669-70.

5. Ostlie DJ, Ponsky TA. Technical options of the laparoscopic pediatric inguinal hernia repair. J Laparoendosc Adv Surg Tech A 2014;24:194-8.

6. Abd-Alrazek M, Alsherbiny H, Mahfouz M, et al. Laparoscopic pediatric inguinal hernia repair: a controlled randomized study. J Pediatr Surg 2017;52:1539-44.

7. Miyake H, Fukumoto K, Yamoto M, et al. Comparison of percutaneous extraperitoneal closure (LPEC) and open repair for pediatric inguinal hernia: experience of a single institution with over 1000 cases. Surg Endosc 2016;30:1466-72.

8. Wang YJ, Zhang QL, Chen L, et al. Laparoscopic Percutaneous Extraperitoneal Internal Ring Closure for Pediatric Inguinal Hernia: 1,142 Cases. J Laparoendosc Adv Surg Tech A 2019;29:845-51.

9. Zhu H, Li J, Peng X, et al. Laparoscopic Percutaneous Extraperitoneal Closure of the Internal Ring in Pediatric Recurrent Inguinal Hernia. J Laparoendosc Adv Surg Tech A 2019;29:1297-301.

10. Zendejas B, Cook DA, Bingener J, et al. Simulationbased mastery learning improves patient outcomes in laparoscopic inguinal hernia repair: a randomized

Cite this article as: Duan S, Zhang P, Lin X, Zheng L. Laparoscopic percutaneous extraperitoneal closure with peritoneum reinforcement repair for pediatric inguinal hernia: a single-center experience with over 2,000 patients. Transl Pediatr 2021;10(5):1317-1323. doi: 10.21037/tp-21-25 controlled trial. Ann Surg 2011;254:502-9; discussion 509-11.

11. Shalaby R, Ismail M, Samaha A, et al. Laparoscopic inguinal hernia repair; experience with 874 children. J Pediatr Surg 2014;49:460-4.

12. Wolak PK, Patkowski D. Laparoscopic inguinal hernia repair in children using the percutaneous internal ring suturing technique - own experience. Wideochir Inne Tech Maloinwazyjne 2014;9:53-8.

13. Miyake H, Fukumoto K, Yamoto M, et al. Risk factors for recurrence and contralateral inguinal hernia after laparoscopic percutaneous extraperitoneal closure for pediatric inguinal hernia. J Pediatr Surg 2017;52:31721. Erratum in: J Pediatr Surg. 2020 Apr;55(4):780. doi: 10.1016/j.jpedsurg.2020.02.022.

14. Lee SR, Choi SB. The efficacy of laparoscopic intracorporeal linear suture technique as a strategy for reducing recurrences in pediatric inguinal hernia. Hernia 2017;21:425-33.

15. Hayashi K, Ishimaru T, Kawashima H. Reoperation After Laparoscopic Inguinal Hernia Repair in Children: A Retrospective Review. J Laparoendosc Adv Surg Tech A 2019;29:1264-70.

16. Grimsby GM, Keays MA, Villanueva C, et al. Nonabsorbable sutures are associated with lower recurrence rates in laparoscopic percutaneous inguinal hernia ligation. J Pediatr Urol 2015;11:275.e1-4.

17. Rao R, Smith M, Markel TA, et al. Modified percutaneous internal ring suturing with peritoneal injury in children: matched comparison to open hernia repair. Surg Endosc 2021;35:854-9.

18. Chu CB, Chen J, Shen YM, et al. Individualized treatment of pediatric inguinal hernia reduces adolescent recurrence rate: an analysis of 3006 cases. Surg Today 2020;50:499-508.

19. Koivusalo AI. A Review of the Incidence, Manifestation, Predisposing Factors, and Management of Recurrent Pediatric Inguinal Hernia. Eur J Pediatr Surg 2017;27:478-83. 